\title{
Femtolensing by Dark Matter Revisited
}

\author{
Andrey Katz, ${ }^{a, b}$ Joachim Kopp,,$^{a, c}$ Sergey Sibiryakov ${ }^{a, d, e}$ and \\ Wei Xue ${ }^{a}$
}

\author{
${ }^{a}$ Theoretical Physics Department, CERN, Geneva, Switzerland \\ ${ }^{b}$ Département de Physique Théorique and Center for Astroparticle Physics (CAP), \\ Université de Genève, 24 quai Ansermet, CH-1211 Genève 4, Switzerland \\ ${ }^{c}$ PRISMA Cluster of Excellence \& Mainz Institute for Theoretical Physics, \\ Johannes Gutenberg University, Staudingerweg 7, 55099 Mainz, Germany \\ ${ }^{d}$ Institute of Physics, Laboratory for Particle Physics and Cosmology (LPPC), \\ Ecole Polytechnique Fédérale de Lausanne (EPFL), CH-1015 Lausanne, Switzerland \\ ${ }^{e}$ Institute for Nuclear Research of the Russian Academy of Sciences, \\ 60th October Anniversary Prospect, 7a, 117312 Moscow, Russia
}

\begin{abstract}
Femtolensing of gamma ray bursts (GRBs) has been put forward as an exciting possibility to probe exotic astrophysical objects with masses below $10^{-13}$ solar masses such as small primordial black holes or ultra-compact dark matter minihalos, made up for instance of QCD axions. In this paper we critically review this idea, properly taking into account the extended nature of the source as well as wave optics effects. We demonstrate that most GRBs are inappropriate for femtolensing searches due to their large sizes. This removes the previous femtolensing bounds on primordial black holes, implying that vast regions of parameter space for primordial black hole dark matter are not robustly constrained. Still, we entertain the possibility that a small fraction of GRBs, characterized by fast variability can have smaller sizes and be useful. However, a large number of such bursts would need to be observed to achieve meaningful constraints. We study the sensitivity of future observations as a function of the number of detected GRBs and of the size of the emission region.
\end{abstract}




\section{Contents}

1 Introduction 1

2 Theory of Femtolensing $\quad 3$

2.1 Point-like Lens and Source, Geometric Optics Regime 3

2.2 Point-like Source and Lens in the Wave Optics Regime 6

2.3 Extended Sources $\quad 7$

2.4 Extended Lens 9

3 Revision of Current Bounds and Sensitivity Estimates $\quad 12$

$\begin{array}{lll}3.1 & \text { GRB models } & 13\end{array}$

$\begin{array}{lll}3.2 & \text { Likelihood Analysis } & 14\end{array}$

$\begin{array}{lll}3.3 & \text { Results } & 16\end{array}$

$\begin{array}{llr}4 & \text { Conclusions } & 18\end{array}$

A The size of the prompt emission region in GRBs 20

\section{Introduction}

The possibility that the dark matter (DM) in the Universe is made up of massive compact objects has been explored since the early days of the DM hunt. These searches have culminated in strong exclusion limits from the MACHO [1], EROS [2], OGLE [3] and HSC/Subaru [4] surveys. Recently, interest in compact DM objects has been revived, with the most popular candidates being primordial black holes (PBHs) [5-7] or ultra-compact mini-halos (UCMHs) that are present for example in axion models [8-11].

While a wide range of experimental observations constrain compact objects with masses above $\sim 10^{-11} M_{\odot}$ (see [12] for a recent review and summary of constraints), bounds on lighter objects are scarcer. Specifically for PBHs, the requirement that they should not evaporate within the lifetime of the Universe by emitting Hawking radiation sets a lower bound on their mass of about $10^{-18} M_{\odot}$. Moreover, the effect of their evaporation on Big Bang Nucleosynthesis and the extragalactic photon background become important already at PBH masses of order $10^{-17} M_{\odot}$, severely constraining this possibility [13]. Other bounds are based on the existence of old neutron stars [14-17] and white dwarfs [18] that would be destroyed if PBHs were abundant. Note that there is some controversy in the literature about the viability of neutron star constraints. While ref. [16] claims to identify a very efficient mechanism of PBH capture by neutron stars, the derivation was questioned in Refs. [17, 19]. On the other hand the constraints of ref. [15] hinge on the assumption that the globular clusters hosting neutron stars are embedded in overdense DM cores. While this assumption is not ruled out, it is also not experimentally supported [20,21]. Indeed, it has even been shown that globular clusters could be formed without any DM overdensities [22, 23].

None of the bounds discussed above applies directly to UCMHs, which below $10^{-11} M_{\odot}$ remain essentially unconstrained.

To probe the mass range between $\sim 10^{-17} M_{\odot}$ and $\sim 10^{-13} M_{\odot}$, femtolensing of distant gamma-ray bursts (GRBs) has been proposed by Gould [24] (see also ref. [25] for a proposal 
along the same lines for somewhat higher masses). The basic idea behind femtolensing is that, while the two images of the GRB created by such a tiny lens cannot be resolved in space or time, their wave fronts will acquire different phases during propagation because of the different path lengths and gravitational potentials they experience. If the phase shift is of order one, one expects to see interference fringes in the frequency spectrum. Since setting meaningful limits with this method requires a sufficient number of GRBs, most works on femtolensing have been purely theoretical until very recently. An early experimental analysis based on BATSE data was presented in ref. [26], but yielded only very weak bounds. The situation has changed dramatically with the advent of Fermi data. There are now dozens of GRBs with reliably measured redshift and frequency spectrum, with hopes to significantly expand this sample in the near future. Fermi data has prompted first observational works on femtolensing of GRBs by black holes: The authors of ref. [27] claim to constrain primordial black holes with masses between $10^{-16}$ and $10^{-14} M_{\odot}$ to contribute no more than $10 \%$ to the total dark matter abundance in the Universe. Although the validity of these bounds has been questioned later because they are based on the assumption that the gamma-ray source is point-like in the lens plain [16, 28] (see also the relevant estimations of [29]), they have been widely accepted as the state of the art.

In this work we critically re-analyze the idea of GRB femtolensing. We will use the techniques of [30], further elaborated in [31]. One of the questions we study is the impact of the non-pointlike nature of GRBs. We will see that most GRBs are too big when projected onto the lens plane to yield meaningful femtolensing limits. Only a small population of GRBs with very fast variability might be suitable for such searches, but even their size is comparable to the Einstein radius of the lenses of interest to us. It must therefore be properly taken into account.

A second question we address is to what extent femtolensing can be used to constrain non-point-like lenses, i.e., objects that extend beyond their Einstein radius. An important example are UCMHs consisting of DM. Indeed, it has been noticed in the 1990s that femtolensing might be relevant for probing UCMHs in the mass range between $10^{-16}$ and $10^{-12}$ solar masses [32]. But this reference still treated the UCMHs as point-like, which is not necessarily in agreement with more recent estimates of their size [33]. Femtolensing of UCMHs has not been re-analyzed since then, in spite of the significant progress that has been made both in our theoretical understanding of compact DM objects and in GRB observations.

Besides accounting for the finite size of the source and lens, we will also emphasize that the geometric optics approximation used in the original proposal [24] breaks down exactly in the mass range where femtolensing appears most promising. In other words, femtolensing in this mass range cannot be described by considering just two images of the source, with properties derived from the geometry of the corresponding lines of sight. Originally, the importance of wave optics effects in femtolensing had been pointed out in [34].

We will find that, especially because of the non-negligible size of the source, the data available to date does not constrain primordial black holes or other compact DM objects. We also compute the sensitivity of hypothetical future surveys as a function of the number of observed GRBs and their transverse size.

The paper is organized as follow. In section 2 we describe the theory of femtolensing, taking into the account all the caveats discussed above: finite size of the source, possibly finite size of the lens, breakdown of the geometric optics approximation. In section 3 , we review the current femtolensing bounds on compact DM objects and show that they do not cover the physically interesting parameter region. We also show projections into the future, arguing 
that a sample of 100 GRBs with transverse size $10^{9} \mathrm{~cm}$ would be needed to exclude DM in the form of PBHs in the mass range from $10^{-16}$ to $5 \times 10^{-15}$ solar masses. We conclude in Sec. 4. Details on the size of the emission region in a GRB are relegated to the Appendix.

\section{Theory of Femtolensing}

In this section we review the general idea of femtolensing and the underlying formalism, and we address several important caveats. We will start by discussing the case of a point-like lens affecting light from a point-like source in the geometric optics approximation. We then introduce one-by-one the wave optics corrections, the effect of an extended source, and the possibility of an extended lens whose size exceeds its would-be Einstein radius. To the best of our knowledge, this study has not been performed before, but is strongly motivated by the appeal of ultra-compact DM miniclusters.

\subsection{Point-like Lens and Source, Geometric Optics Regime}

The basic femtolensing scenario, put forward in [24], is based on the assumption that a gamma ray emitted by a point-like source with a non-zero impact parameter with respect to the lens-observer axis, is split by the lens into two rays, each of which is delayed with respect to the unlensed case by some time shift $\Delta t_{i}(i=1,2)$. This corresponds to a phase shift of $\Delta \phi_{i} \equiv \omega \Delta t_{i}$, where $\omega$ is the angular frequency of the photons. If the two images cannot be resolved in space and time, the two rays will interfere, producing characteristic fringes in the spectrum.

In the thin lens approximation the time delay is given by [35]

$$
\Delta t=\frac{1}{c} \frac{D_{L} D_{S}}{D_{L S}}\left(1+z_{L}\right)\left(\frac{|\vec{\theta}-\vec{\beta}|^{2}}{2}-\psi(\vec{\theta})\right) .
$$

Here $D_{L}, D_{S}$, and $D_{L S}$ are the angular diameter distances between the observer and the lens, the observer and the source, and the lens and the source, respectively. The redshift of the lens is denoted $z_{L}$, while $\beta$ is the angle under which the observer would see the source in the absence of a lens, and $\theta$ is the angle under which the observer sees a given point in the lens plane. The function $\psi(\theta)$ is the lensing potential which is related to the density profile $\rho(r)$ of the lens by the Poisson equation

$$
\nabla^{2} \psi(\theta) \equiv \frac{1}{\theta} \frac{\partial}{\partial \theta}\left(\theta \frac{\partial \psi}{\partial \theta}\right)=\frac{8 \pi G}{c^{2}} \frac{D_{L S} D_{L}}{D_{S}} \int_{-\infty}^{\infty} d \xi \rho\left(\sqrt{\left(D_{L} \theta\right)^{2}+\xi^{2}}\right)
$$

Here, the integral runs along the line of sight, and $\nabla^{2}$ is the two-dimensional Laplace operator, which we express in polar coordinates, with $\theta$ being the radial direction. We have also assumed a spherically symmetric lens. For a point-like lens of mass $M,{ }^{1}$

$$
\psi(\theta)=\theta_{E}^{2} \log \theta \quad \text { (point-like lens), }
$$

\footnotetext{
${ }^{1}$ Note that the lensing potential is defined by eq. (2.2) only up to an additive constant. We will ignore this constant here, which implies that the expression for the time delay, eq. (2.1), is applicable only for calculating the time difference between different paths, but does nor necessary reflect the absolute time delay along the path.
} 
where

$$
\theta_{E} \equiv\left(\frac{4 G M}{c^{2}} \frac{D_{L S}}{D_{S} D_{L}}\right)^{1 / 2}
$$

is the Einstein angle (i.e. the Einstein radius $R_{E}$ divided by $D_{L}$ ). On top of being a convenient definition, the Einstein angle has a well defined physical meaning: in the geometric optics approximation, it is the size of the Einstein ring produced by a point-like source aligned with the observer-lens line of sight $(\beta=0)$. For the more general density profiles that we will discuss in section 2.4 the location of the ring will be different from $\theta_{E}$ and will be called $\theta_{0}$. For point-like masses, the two values coincide.

In geometric optics, Fermat's principle stipulates that the images will be seen under those angles $\theta$ for which $\Delta t$ is stationary. For point-like lens and source, this requirement leads to the lens equation ${ }^{2}$

$$
\theta-\beta=\frac{\theta_{E}^{2}}{\theta}
$$

Lensing is typically observable when $\theta \sim \theta_{E}$ (or, more generally, when $\theta \sim \theta_{0}$ for non-pointlike lenses), otherwise one of the images becomes extremely faint. This shows that both terms in eq. (2.1) scale as $\theta_{E}$ up to order-one factors. The time delay thus depends only on the mass of the lens and is practically insensitive to the distances either to the source or to the lens: $\Delta t \sim 4 G M / c^{3}=2 R_{s} / c$, where $R_{s}$ is the Schwarzschild radius of the lens. Assuming that the source emits gamma rays in the 10 to $1000 \mathrm{keV}$ range, we see that order-one phase shifts occur for lens masses between $10^{-17}$ and $10^{-14}$ solar masses. For lensing at cosmological distances $\sim 1 \mathrm{Gpc}$ the corresponding Einstein angles fall in the femto-arc-second range, which explains the term "femtolensing".

The solutions to the lens equation (2.5) are

$$
\theta_{ \pm}=\frac{1}{2}\left(\beta \pm \sqrt{\beta^{2}+4 \theta_{E}^{2}}\right)
$$

The magnifications of the two images are given by [35]

$$
\mu_{ \pm}=\frac{y^{2}+2}{2 y \sqrt{y^{2}+4}} \pm \frac{1}{2}
$$

where we have defined

$$
y \equiv \beta / \theta_{E} .
$$

Taking into account the phase shift between the images, the total intensity is proportional to $[31]$

$$
\mu=\frac{y^{2}+2}{y \sqrt{y^{2}+4}}+\frac{2}{y \sqrt{y^{2}+4}} \sin \left(\Omega\left[\frac{y \sqrt{y^{2}+4}}{2}+\log \left|\frac{y+\sqrt{y^{2}+4}}{y-\sqrt{y^{2}+4}}\right|\right]\right),
$$

where we have introduced the dimensionless frequency as

$$
\Omega \equiv \frac{1}{c} \frac{D_{S} D_{L}}{D_{L S}} \theta_{0}^{2}\left(1+z_{L}\right) \omega \equiv \Delta t_{0} \omega .
$$

\footnotetext{
${ }^{2}$ The generic lens equation is $\vec{\theta}-\vec{\beta}=\vec{\nabla} \psi(\vec{\theta})$.
} 
Here we have also introduced a new quantity $\Delta t_{0}$ which is a typical time delay between different images in the geometric optics picture. Note that this definition of the dimensionless frequency is completely generic and we are going to use it also for extended mass distributions in section 2.4. For point-like lenses, however, eq. (2.10) simplifies to

$$
\Omega \equiv \frac{4 G M\left(1+z_{L}\right)}{c^{3}} \omega .
$$

Equation (2.9) clearly depends on the photon energy $\omega$ and varies between a maximum and minimum value as a function of $\omega$. Therefore a signal of femtolensing would be an oscillatory pattern of interference fringes in the otherwise smooth GRB spectrum.

Let us list the conditions required for the validity of the above formulas.

1. The limit of geometric optics should be applicable. ${ }^{3}$ This is the case as long as

$$
\omega \Delta t_{0} \gg 1
$$

(see section 2.2 for details). In the case of a point-like lens, this reduces (up to a factor $\left.4 \pi\left(1+z_{L}\right)\right)$ to the condition that the photon wave length should be much smaller than the Schwarzschild radius $R_{s}$ of the lens. Note, however, that this does not mean that the Schwarzschild radius can be interpreted as the effective radius of the lens, because the typical deflection distance of the photons is $R_{E}$ rather than $R_{s}$. Moreover, the relation eq. (2.12) to the Schwarzschild radius is something very peculiar to point-like lenses and does not hold for more general mass distributions. A simple estimate shows that the inequality (2.12) breaks down for $M \lesssim 10^{-15} M_{\odot}$ for a gamma ray energy of $E \sim 100 \mathrm{keV}$, essentially invalidating the geometric optics approach in most of the parameter space that is relevant for femtolensing. The breakdown of the geometric optics approximation does not imply the absence of an observable femtolensing effect. It does, however, mean that a full wave optics computation is necessary (see section 2.2).

2. The source should be point-like in the plane of the lens. The signals from different points of the source add up incoherently, and the condition that this does not wash out the interference fringes is

$$
\sigma_{y} \lesssim \frac{1}{\Omega}
$$

where $\sigma_{y}$ is the angular size of the source normalized to $\theta_{0}$. This condition can be easily derived from eq. (2.9). Since interference fringes can only be observed if $\Omega \gtrsim 1$, condition (2.13) also implies that the size of the source projected on the lens plane should be smaller than the Einstein radius for point-like lenses ( $\theta_{0}$ for extended lenses). As we will see later, this condition is never fully satisfied for femtolensing of GRBs. At best, the projected size of the source can be comparable to the Einstein radius, so that finite size effects can never be neglected. Note also that the constraint on the size of the source is stricter for higher frequencies/energies. Therefore, as the size of the source increases, the interference fringes will first disappear at high frequencies.

3. The source emission must be coherent over timescale of the time delay, eq. (2.1). Usually this condition is easily satisfied because it merely requires a detector with sufficient

\footnotetext{
${ }^{3}$ We are grateful to Juan Garcia-Bellido for pointing out to us the importance of wave optics effects.
} 


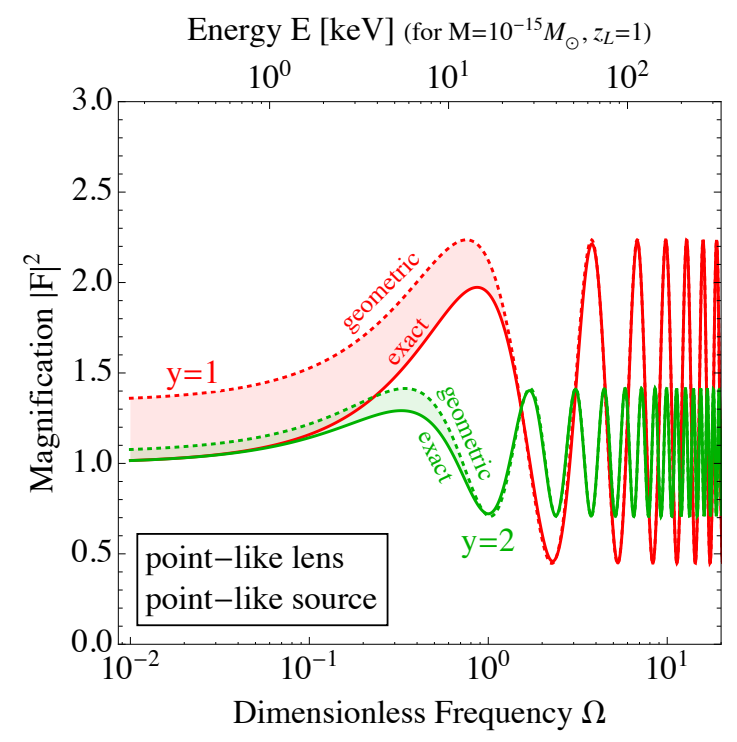

Figure 1. Comparison of the interference picture in the geometric optics approximation (dashed) and the full wave optics results (solid) in the case of a point-like source and a point-like lens. Wave effects strongly impact the magnification of the signal at low energies, while at higher energies, geometric optics provides a good approximation. The magnification is plotted against the dimensionless frequency $\Omega$ defined in Eq. (2.11).

energy resolution. Typically, we are interested in scenarios where $\omega \Delta t_{0} \sim 1$. Given that any reasonable detector has an energy (or frequency) uncertainty $\delta \omega \ll \omega$, the Heisenberg uncertainty principle tells us that the coherence time $\delta t \sim \delta \omega^{-1}$ is much larger than $\Delta t_{0}$.

Because the conditions (1) and (2) are never strictly satisfied, we are forced to relax some of the approximations made above. We will do so in the following subsections.

\subsection{Point-like Source and Lens in the Wave Optics Regime}

Gravitational lensing outside the geometric optics regime is not an unusual scenario. For example, it has been mentioned in [36] that the microlensing measurements of HSC Subaru are partially in the physical (wave) optics regime, invalidating the constraints on primordial black holes published in ref. [4] for masses below $10^{-11} M_{\odot}$. To the best of our knowledge, the correct interpretation of the HSC constraints in this mass range is still missing in the literature. Also, practically all discussion of lensing of gravitational waves includes wave optics effects [37] (for recent related works see e.g. [38-40]). Finally, it was noticed already in the 1990s that the geometric optics approximation is typically violated in femtolensing [34].

In the physical optics regime, lensing is characterized by a magnification function $F(\vec{y} ; \omega)$, which is defined as the ratio $\phi_{L} / \phi$, where $\phi_{L}$ and $\phi$ are the electromagnetic wave amplitudes with and without lensing, respectively. The magnification of the signal intensity is therefore

$$
\mu=|F|^{2},
$$

The magnification function is given by [41]

$$
F(\vec{y} ; \omega)=\frac{\Omega}{2 \pi i} \int d^{2} \vec{x} e^{i \omega \Delta t(\vec{x}, \vec{y})} .
$$


where the dimensionless frequency $\Omega$ has been defined in eq. (2.10), $x \equiv \theta / \theta_{0}$, and the time delay $\Delta t(\vec{x}, \vec{y})$ is taken from eq. (2.1). The integral thus runs over the lens plane, with each point in the plane contributing to $F(\vec{y}, \omega)$. For a point-like lens like a black hole, the integral can be evaluated analytically, leading to

$$
F(y, \Omega)_{\mathrm{BH}}=e^{i \Omega|\vec{y}|^{2} / 2}\left(-\frac{i \Omega}{2}\right)^{i \Omega / 2} \Gamma\left(1-\frac{i \Omega}{2}\right) L_{-1+\frac{i \Omega}{2}}\left(-\frac{i|\vec{y}|^{2} \Omega}{2}\right)
$$

where $L_{n}$ is the $n$-th order Laguerre polynomial. For an extended lens, analytic expressions in general do not exist, so the integral in eq. (2.15) must be evaluated numerically.

The geometric optics regime corresponds to the saddle point approximation of (2.15): as discussed in section 2.1, the lens equation (2.5) is obtained by imposing the stationary point condition $\vec{\nabla}_{x} \Delta t=0$. Also the magnification $\mu$ in the geometric optics limits, eq. (2.7), can be derived from the saddle point method. However, the saddle point method only provides a good approximation to the full integral if the phase is large, which explains why geometric optics only works if $\omega \Delta t \gg 1$.

In Fig. 1, we compare the intensity calculated using the exact wave optics expression, eq. (2.15), to the one obtained in the geometric optics approximation, eq. (2.9). We see that the geometric optics approximation overestimates the amplitude of the first fringe in the spectrum, while at higher photon energies, it is applicable. We will see in section 2.4 that the effects of wave optics are more important for extended lens profiles.

\section{$2.3 \quad$ Extended Sources}

As argued above, interference patterns due to femtolensing are only observable if the size of the source projected onto the lens plane is not much larger than the Einstein radius of the lens (assumed to be point-like in this subsection). In other words, we require that

$$
\sigma_{y} \equiv \frac{a_{S}}{D_{S} \theta_{E}} \ll 1
$$

where $a_{S}$ is the actual (unprojected) transverse size of the emission region. Most GRBs have an $\mathcal{O}(1)$ redshift, corresponding to $D_{S} \sim$ Gpc. Therefore a lens of mass $M \sim 10^{-15} M_{\odot}$ that is also located at $z_{L} \sim \mathcal{O}(1)$ has an Einstein radius of order $R_{E} \sim 10^{9} \mathrm{~cm}$. The Einstein radius of lighter lenses will be even smaller. Unfortunately, the emission size of most GRBs is much larger than this $[29,42]$.

As discussed in appendix A, the majority of GRBs are likely to have transverse sizes of about $a_{S} \sim 10^{11} \mathrm{~cm}$, which is two orders of magnitude larger than required for observable femtolensing. Still, the possibility that a small fraction of GRBs have much smaller sizes $a_{S} \lesssim 10^{9} \mathrm{~cm}$ is not excluded. We will entertain this possibility and study the dependence of the femtolensing signal on the size of the source. Hopefully, future developments in GRB modeling, perhaps on a case-by-case basis, will lead to more precise estimates of their sizes than are currently available, and will allow us to cherry-pick events suitable for femtolensing. Note that, if such GRBs exist, they will be characterized by very fast intrinsic variability at time scales ${ }^{4} t_{\mathrm{var}} \lesssim 0.5 \times 10^{-3}$ sec.

\footnotetext{
${ }^{4}$ We leave aside the question whether such variability time scales can be measured with existing instruments, as the main focus of our study are future observations.
} 


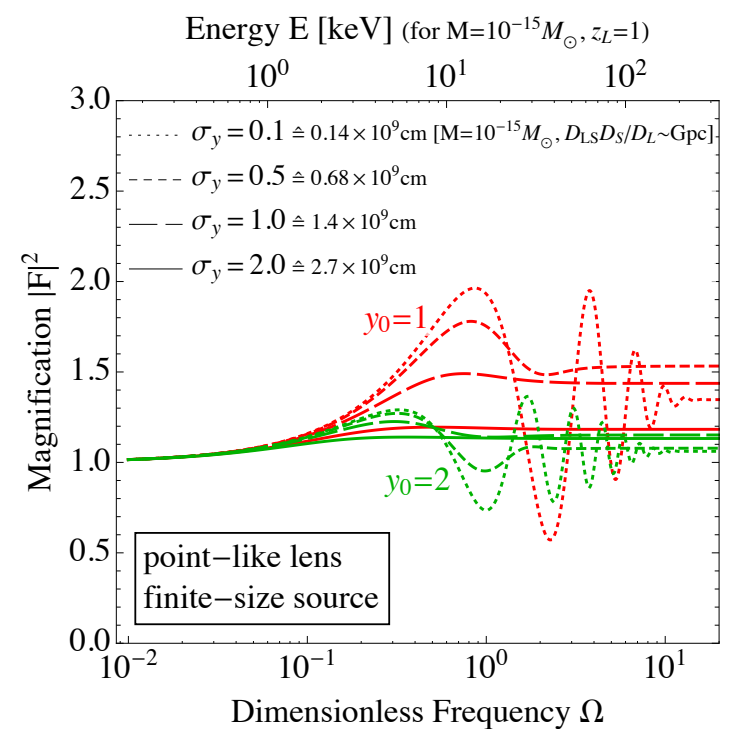

Figure 2. Dependence of magnification on the size of the source. Here $\sigma_{y}$ is the angular size of the source in units of the Einstein angle, see eq. (2.17). The dimensionless frequency $\Omega$ is defined in eq. (2.11). The lens is assumed to be point-like.

To take the finite size of the source into account, we follow the formalism of ref. [30]. The observed magnification is then

$$
\bar{\mu}=\frac{\int d^{2} y W\left(\vec{y} ; \sigma_{y}\right) \mu(\vec{y} ; \Omega)}{\int d^{2} y W\left(\vec{y} ; \sigma_{y}\right)},
$$

where $W\left(y ; \sigma_{y}\right)$ is a window function that describes the intensity profile of the emission and $\mu(\vec{y} ; \Omega)$ is the magnification for a point-like source, see eqs. $(2.14),(2.15)$. In principle $W(\vec{y})$ can be any well-behaved function that acts as a mask of size $\sigma_{y}$. We choose it to be a Gaussian,

$$
W\left(y ; \sigma_{y}\right)=e^{-\left|\vec{y}-\vec{y}_{0}\right|^{2} / 2 \sigma_{y}^{2}} .
$$

Hereafter, we will use $\vec{y}_{0}$ to denote the location of the center of the emission. For Gaussian $W\left(y ; \sigma_{y}\right)$ and a radially symmetric lens, the weighted magnification reads

$$
\bar{\mu}=\frac{e^{-y_{0}^{2} / 2 \sigma_{y}^{2}}}{\sigma_{y}^{2}} \int_{0}^{\infty} d y y e^{-y^{2} / 2 \sigma_{y}^{2}} I_{0}\left(\frac{y_{0} y}{\sigma_{y}^{2}}\right) \mu(y ; \Omega)
$$

where $I_{0}(x)$ is the modified Bessel function of the first kind.

We illustrate the effect of the non-zero source size in fig. 2. Clearly, the effect is mild for $\sigma_{y} \ll 1$, however even for these values of the emission size the oscillations in energy space are damped at high frequencies. As the emission size grows, oscillations are damped more strongly, until they eventually disappear at $\sigma_{y} \sim 1$.

Interestingly, we observe that, even at large emission size $\sigma_{y} \gtrsim 1$, the asymptotic $\bar{\mu}$ at high frequency is larger than the value of $\bar{\mu}$ at low frequency. That is, even though the interference fringes are washed out, a smooth step-like feature survives. This can be understood as follows. At $\Omega \rightarrow \infty$ the point-source magnification $\mu(y ; \Omega)$ is well described 
by the geometric optics expression (2.9). It quickly oscillates as a function of $y$ and can be replaced in the integral (2.20) by its mean value,

$$
\mu(y ; \Omega) \mapsto \frac{y^{2}+2}{y \sqrt{y^{2}+4}} .
$$

Thus, in the high frequency limit the weighted magnification reads

$$
\lim _{\Omega \rightarrow \infty} \bar{\mu}=\frac{e^{-y_{0}^{2} / 2 \sigma_{y}^{2}}}{\sigma_{y}^{2}} \int_{0}^{\infty} d y \frac{y^{2}+2}{\sqrt{y^{2}+4}} e^{-y^{2} / 2 \sigma_{y}^{2}} I_{0}\left(\frac{y_{0} y}{\sigma_{y}^{2}}\right)>1 .
$$

On the other hand, in the limit $\Omega \rightarrow 0$ the geometric optics approximation is not applicable and the wave optics calculation leads to $\lim _{\Omega \rightarrow 0} \bar{\mu}=1$. The difference between the two limiting values leads to the step-like feature in the weighted magnification function at low dimensionless frequencies observed in fig. 2. Of course, in the absence of oscillatory fringes, one probably cannot rely on this feature to establish that a given GRB spectrum has been lensed. On the other hand, a non-observation of such feature can potentially be used to exclude lensing. Since our proposal to perform an exclusion based on the absence of this feature is rather speculative, we will not use it in estimating the future reach in section 3. However, if proven possible, this would imply that even $\gamma$-ray sources as big as $10^{10} \mathrm{~cm}$ might be used to exclude femtolensing.

\subsection{Extended Lens}

We finally analyze the effects of a non-point-like lens, namely a lens whose extent is larger than its would-be Einstein radius. A notable example of such lenses would be ultracompact minihalos (UCMHs) composed of DM. These are predicted, for instance, in scenarios that involve DM in the form of QCD axions if the Peccei-Quinn phase transition happens after inflation. In this case, overdensities that arise after the phase transition due to different initial values of the axion field in different Hubble patches collapse into UCMHs around matterradiation equality $[8,10,33]$. The mass and radius estimates of UCMHs vary widely in the literature. For example, an average value for the mass of the QCD axion miniclusters as small as $10^{-14} M_{\odot}$ was suggested in [43]. This is two orders of magnitude lighter than the previous estimates of $[28,32,44,45]$. Even bigger masses were suggested in [46, 47]. Although resolving these discrepancies is beyond the scope of this work, some part of the suggested parameter space is likely to be within the reach of femtolensing searches, as has been first pointed out in [32]. UCMHs are not unique to QCD axions and can be formed also in many other models with axion-like particles [11]. The parameter space explored in ref. [11] is vast, and some of it is definitely accessible to femtolensing, in particular axion-like particles with temperature-independent $(n=0)$ masses of $\sim 10^{-3}-10^{-5} \mathrm{eV}$.

Another parameter subject to significant uncertainties is the radius of the UCMHs. However, what is important for our discussion is that it is most likely bigger than its wouldbe Einstein radius. For instance, for the QCD axion, assuming miniclusters are spherically symmetric, refs. [32, 33, 44] give,

$$
R_{\mathrm{UCMH}} \simeq \frac{3 \times 10^{12} \mathrm{~cm}}{\Phi(1+\Phi)^{1 / 3}}\left(\frac{M}{10^{-12} M_{\odot}}\right)^{1 / 3}
$$

where $\Phi$ is the initial axion density contrast. Most miniclusters will have $\Phi \lesssim 10$ [32], so their radius according to eq. (2.23) is almost two orders of magnitude larger than their 
corresponding Einstein radius. Only for extremely dense miniclusters with $\Phi \gtrsim 100$, the size and the Einstein radius become comparable. It is evident from these estimates that one cannot neglect the extent of the UCMHs in the lensing problem.

Finally, there is no agreement in the literature on the density profile of UCMHs, which may also depend on the mechanism by which they form in the early Universe. Some scenarios suggest rather steep profiles. For example, the self-similar infall scenario motivates a density profile of the form $\rho(r) \propto r^{-9 / 4}[48,49]$. This profile has been confirmed in N-body simulations [33, 50, 51], but its relevance to UCMHs was later questioned in [52]. The latter paper advocates more shallow profiles, $\rho(r) \propto r^{-3 / 2}$, or the Navarro-Frenk-White (NFW) profile which scales as $\rho(r) \propto r^{-1}$ at small radii. Given this uncertainty, we prefer to be agnostic about the precise shape of the UCMH profile. Rather we will assume that its inner part is described by a generic power law cusp,

$$
\rho(r)=\rho_{0}\left(\frac{r}{r_{0}}\right)^{-\delta}, \quad \delta<3
$$

where $r_{0}$ characterizes the size of the inner part of the $\mathrm{UCMH}$ and $\rho_{0}$ is the density at that distance. ${ }^{5}$ It is convenient to introduce the mass enclosed within radius $r_{0}$, which we will call the "cusp mass",

$$
M_{\text {cusp }}=\frac{4 \pi}{3-\delta} \rho_{0} r_{0}^{3} .
$$

Note that the total mass of UCMHs can, in general, be bigger than $M_{\text {cusp }}$ and can, in fact, even be formally divergent. The Einstein radius corresponding to $M_{\text {cusp }}$ is

$$
R_{E, \text { cusp }}=\left(\frac{4 G M_{\text {cusp }}}{c^{2}} \frac{D_{L S} D_{L}}{D_{S}}\right)^{1 / 2} .
$$

It is this radius that we mean when referring to the Einstein radius of the UCMH. We remind the reader that for extended lenses, the Einstein radius does not have an immediate physical interpretation. We assume $R_{E \text {, cusp }}<r_{0}$, such that the cusp cannot be treated as point-like for the purposes of gravitational lensing.

It is easy to see that only profiles with $\delta>1$ can be relevant for femtolensing. Indeed, the mass enclosed within a radius $r$ grows as $M(r) \propto r^{3-\delta}$ and the corresponding Einstein radius scales as $R_{E}(r) \propto r^{(3-\delta) / 2}$. If $\delta \leq 1$, any part of the lens is bigger than its Einstein radius, $R_{E}(r)<r$, so no multiple images and thus no femtolensing can arise. On the other hand, for $\delta>1$, the central part of the cusp happens to be within its Einstein radius. Thus, it acts qualitatively similar to a point-like lens leading to appearance of multiple images.

We now study the case $\delta>1$ in more detail. Following eq. (2.2), the lensing potential corresponding to the density profile eq. (2.24) is

$$
\psi(\theta)=\frac{\theta_{0}^{2}}{3-\delta}\left(\frac{\theta}{\theta_{0}}\right)^{3-\delta}
$$

\footnotetext{
${ }^{5}$ Note that some steep profiles tend to develop a core at the center of the distribution. In particular, the self-similar radial infall profile corresponding to $\delta=9 / 4$ cannot be a valid approximation all the way down to $r=0$ (see e.g. [53] for an approach to estimate the size of the core). While we will for simplicity neglect the presence of the core in the sensitivity studies presented in this paper, it should be taken into account when analyzing real data.
} 
where we have defined

$$
\theta_{0}=\kappa(\delta) \frac{R_{E, \text { cusp }}}{D_{L}}\left(\frac{R_{E, \text { cusp }}}{r_{0}}\right)^{\frac{3-\delta}{\delta-1}}, \quad \kappa(\delta) \equiv\left(\frac{\sqrt{\pi} \Gamma((\delta-1) / 2)}{2 \Gamma(\delta / 2)}\right)^{\frac{1}{\delta-1}} .
$$

The angle $\theta_{0}$ is an analog of the Einstein angle for extended lenses. In the geometric optics approximation it coincides with the angular size of the Einstein ring produced by a pointlike source aligned with the center of the lens $(\beta=0$ in the notations of section 2.1). For misaligned sources $(\beta \neq 0), \theta_{0}$ sets the characteristic distance between their multiple images. The characteristic time delay $\Delta t_{0}$ is defined in (2.10). Note that $\theta_{0}$ is parametrically smaller than the naive Einstein angle $R_{E \text {, cusp }} / D_{L}$.

For our subsequent discussion, we need to distinguish between the cases $\delta<2$ and $\delta>2$. For $1<\delta<2$ and small enough $\beta$, the lens equation $\vec{\theta}-\nabla \psi(\vec{\theta})=\vec{\beta}$ has three solutions corresponding to three images in the geometric optics approximation. Above a certain critical value $\beta_{\mathrm{cr}}=C(\delta) \theta_{0}$, where $C(\delta)$ is an order-one coefficient, two of the images disappear and only a single one remains. In other words, the $\operatorname{ring} \beta=\beta_{\mathrm{cr}}$ is a caustic. Thus, the appearance of interference fringes characteristic for femtolensing is possible only if the source is close enough to the line of sight passing through the center of the lens. Then, the existence of more than two images will in general lead to a complicated interference pattern (cf. ref. [34]).

The properties of a lens with $2<\delta<3$, including the self-similar infall profile with $\delta=$ $9 / 4$, are closer to those of point-like objects like PBHs. In this case the lens equation always has two solutions corresponding to two images in the geometric optics approximation. This will give rise to the characteristic sinusoidal dependence of the magnification on frequency. The caustic shrinks to the point $\beta=0$, at which the two images turn into an Einstein ring of angular radius $\theta_{0} \cdot{ }^{6}$

Wave optics effects quantitatively modify the geometric optics results at low frequencies, but do not lead to qualitative changes. We have computed the magnification numerically for the case $\delta=9 / 4$ using eqs. (2.14) and (2.15). The results are shown in fig. 3, and are also compared to the geometric optics approximation. As expected from the above discussion, the behavior of the curves is similar to the case of a point-like lens, but it nevertheless differs in some important details. First, wave optics corrections remain sizeable up to higher energies. Even more important, for impact parameters $y \equiv \beta / \theta_{0}>1$, we find that the geometric optics approximation significantly underestimates the amplitude over the first 5-7 periods of the oscillation. As the impact parameter grows, the amplitude of the oscillations falls off more slowly towards higher frequency than for a point-like lens, increasing the lensing probability for UCMHs compared to PBHs.

Comparing figs. 1 and 3, we observe that the interference fringes for UCMHs are shifted to higher energies compared to the case of PBHs. This can be understood by noting from eq. (2.2) that a photon passing the lens at a distance $\theta D_{L}$ is affected by DM particles residing at radii less than $\theta D_{L}$. The characteristic distance at which lensed photons pass an UCMH is $\theta_{0} D_{L}$ (see eq. (2.28)). So if we call the mass contained within this radius $m\left(\theta_{0}\right)$, we expect the interference pattern for an UCMH to be comparable to the one for a PBH with mass $m\left(\theta_{0}\right)$ (modulo obvious differences in the lensing potential and the reduced deflection angle). We can thus estimate that the interference fringes in fig. $3(\delta=9 / 4)$ should be shifted to

\footnotetext{
${ }^{6}$ The case isothermal sphere profile with $\delta=2$ lies at the boundary between the two regimes discussed here: there are two lensed images for $\beta<\theta_{0}$ and a single image for $\beta>\theta_{0}$.
} 


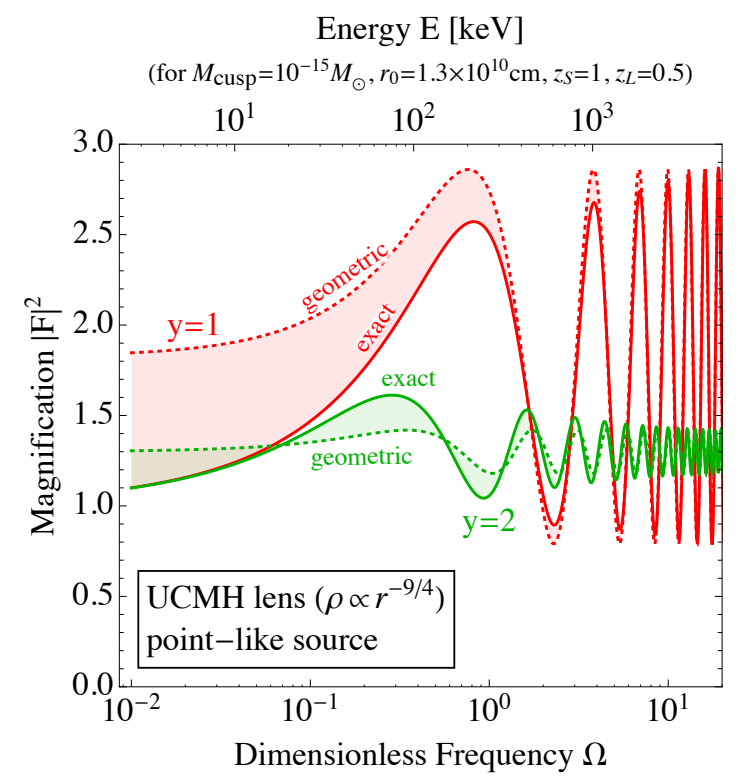

Figure 3. True magnification vs. geometric optics approximation for the self-similar infall profile $\rho(r) \sim r^{-9 / 4}$ as a function of the dimensionless frequency $\Omega=\omega \Delta t_{0}$, see eq. (2.10). We see that, for impact parameter $y \equiv \beta / \theta_{0}=1$, the picture is qualitatively quite similar to that for a point-like mass. At larger $y$, however, the geometric optics approximation significantly underestimates the magnitude of the lensing effect in a wide frequency range.

higher energies by a factor

$$
\frac{m\left(\theta_{0}\right)}{M_{\text {cusp }}}=\left(\frac{\theta_{0} D_{L}}{r_{0}}\right)^{3 / 4}
$$

compared to the ones in fig. 1 . For $z_{S}=1$ and $z_{L}=0.5$, this becomes

$$
\frac{m\left(\theta_{0}\right)}{M_{\text {cusp }}}=0.037 \times\left(\frac{1.3 \times 10^{10} \mathrm{~cm}}{r_{0}}\right)^{6 / 5}\left(\frac{M_{\text {cusp }}}{10^{-15} M_{\odot}}\right)^{3 / 5} .
$$

This estimate is indeed in good agreement with the shift observed in the plots.

\section{Revision of Current Bounds and Sensitivity Estimates}

In this section, we will revisit the femtolensing bounds from ref. [27] by considering wave optics corrections (see section 2.2) as well as the non-pointlike nature of the GRB sources (see section 2.3). From Fig 1 we expect that wave optics effects will modify femtolensing bounds on point-like masses by at most a few tens of per cent compared to the geometric optics approximation. The finite size of the sources, however, is expected to lead to much more dramatic modifications. As the size $a_{S}$ of the emission region in a GRB is very uncertain (see appendix A), we will investigate the dependence of our results on $a_{S}$. We will find that only if $a_{S} \lesssim 10^{8} \mathrm{~cm}$, current data is able to set meaningful limits. This is true for point-like lenses such as primordial black holes, but also for extended lenses like axion miniclusters. Unfortunately the assumption $a_{S} \lesssim 10^{8} \mathrm{~cm}$ is not realistic. 
We will establish these conclusions by investigating the sensitivity of current data, i.e. by working with simulated GRB data rather than real data. This will make our analysis more transparent, and our conclusion will be that current data is not sensitive to DM femtolensing yet. Therefore it is not necessary to go beyond a sensitivity study, and we will therefore not analyze actual Fermi GRBs data. We will, however, extrapolate our results into the future and estimate how many well observed GRBs would be needed for femtolensing to become a competitive player in the hunt for compact DM objects.

In the following, we will first describe how we model GRB spectra (section 3.1). We will then describe the statistical methods we use (section 3.2) and discuss the resulting sensitivity estimates for PBHs and UCMHs (section 3.3).

\subsection{GRB models}

We simulate "data" based on a phenomenological model for the unlensed GRB spectrum. In particular, we use Band's model (BAND) [54] as our baseline scenario, but we have also studied a broken power law model (BKN) as well as a simple power law model with an exponential cutoff as a cross check.

The BAND model has four free parameters: an amplitude $A$, two spectral indices $\alpha_{1}$ (low energy) and $\alpha_{2}$ (high energy), and an energy scale $E_{0}$. In terms of these parameters, the spectrum as a function of energy $E$ is given by

$$
f_{\mathrm{BAND}}(E)= \begin{cases}A\left(E / E_{0}\right)^{\alpha_{1}} \exp \left(-E / E_{0}\right) & \text { if } E \leq\left(\alpha_{1}-\alpha_{2}\right) E_{0} \\ A\left[\left(\alpha_{1}-\alpha_{2}\right)\right]^{\alpha_{1}-\alpha_{2}}\left(E / E_{0}\right)^{\alpha_{2}} \exp \left(\alpha_{2}-\alpha_{1}\right) & \text { otherwise }\end{cases}
$$

In our sensitivity studies, we choose $A=0.15$ counts $\mathrm{sec}^{-1} \mathrm{~cm}^{-2} \mathrm{keV}^{-1}, E_{0}=160 \mathrm{keV}$, $\alpha_{1}=-0.9$, and $\alpha_{2}=-2.5$. These parameters are based on a fit to Fermi GBM data on GRB 090424 [55], and we have normalized the spectrum to 5000 photons in the energy range from $8 \mathrm{keV}$ to $550 \mathrm{keV}$ on which we will focus in our analysis. This normalization is roughly based on the sample of high quality events that the Fermi GBM can collect for a typical short GRB at $z_{S}=1$. We treat the detector's effective area as a constant, $100 \mathrm{~cm}^{2}$, and we assume the GRB to last $1 \mathrm{sec}$. These are typical values for short GRBs, which may appear more interesting for femtolensing because of the arguments given in appendix A that indicate that the size of the emission region, $a_{S}$, tends to be smaller for short GRBs. Nonetheless, given the possibility of a broad distribution of $a_{S}$ for long GRBs, their larger abundance and higher redshift, it is unclear which type of GRB will ultimately offer the best sensitivity.

The BKN model has four free parameters as well: an amplitude $A$, a characteristic energy $E_{0}$, and two spectral indices $\alpha_{1}, \alpha_{2}$ :

$$
f_{\mathrm{BKN}}(E)=\left\{\begin{array}{ll}
A\left(\frac{E}{E_{0}}\right)^{-\alpha_{1}} & \text { if } E \leq E_{0} \\
A\left(\frac{E}{E_{0}}\right)^{-\alpha_{2}} & \text { if } E>E_{0}
\end{array} .\right.
$$

The benchmark parameters for the BKN model are $A=0.099$ counts $\mathrm{sec}^{-1} \mathrm{~cm}^{-2} \mathrm{keV}^{-1}$, $E_{0}=160 \mathrm{keV}, \alpha_{1}=-0.9$, and $\alpha_{2}=-2.5$.

The power law model with exponential cutoff has only three free parameters: the amplitude $A$, a spectral index $\alpha$, and an energy scale $E_{0}$ :

$$
f_{\text {power-exp }}=A E^{\alpha} \exp \left(-E / E_{0}\right) .
$$


The benchmark parameters for this model are $A=0.16$ counts $\mathrm{sec}^{-1} \mathrm{~cm}^{-2} \mathrm{keV}^{-1}, E_{0}=$ $150 \mathrm{keV}$, and $\alpha=-0.92$. This choice leads to the same normalization as for the BAND model. $^{7}$

We choose energy-dependent bin sizes of $2 \delta E$, where we assume

$$
\frac{\delta E}{E}=\sqrt{\left(\frac{0.05}{\sqrt{E / \mathrm{keV}}}\right)^{2}+(0.05)^{2}} .
$$

This resolution is better than that of the Fermi GBM instrument [57] because we will mainly be interested in the sensitivity of future observatories.

In fig. 4, we show a simulated data set based on these assumptions and using the BAND model. We see that the magnitude of the femtolensing effect depends crucially on the size of the source. If $a_{S}=10^{8} \mathrm{~cm}$, a pronounced interference pattern emerges. However already for $a_{S} \sim 10^{9} \mathrm{~cm}$ - which is still rather optimistic - it is already quite difficult to extract a clear signal, even if the lens is located at very low redshift $z_{L}=0.05$, so that the projected size of the source in the lens plane is reduced compared to the case $z_{L} \sim 1$. Had we chosen the same $z_{L}$ for the orange curve as for the blue one $\left(a_{S} \sim 10^{8} \mathrm{~cm}\right)$, the amplitude of the femtolensing wiggles would be comparable to the error bars on the data or even smaller. Similarly, for larger $a_{S} \sim 10^{10} \mathrm{~cm}$, the femtolensing effect disappears. Parenthetically we notice that in order to get the realistic pattern, one should further convolve it with the detector response function (see the black dotted lines). We checked explicitly, that the resolution that we have assumed in eq. (3.4) does not change qualitatively any of the curves that we show, the current Fermi resolution wipes out the effect completely for both lines. This further reinforces our point that current Fermi observation cannot even be sensitive to the emission size as small as $a_{s}=10^{8} \mathrm{~cm}$ (which is extremely optimistic), and better resolutions of future experiments would be needed to make any further progress.

\subsection{Likelihood Analysis}

To quantitatively analyze the simulated data and compare to femtolensing predictions, we define a log-likelihood function ${ }^{8}$

$$
-2 \log L_{0}\left(\vec{\mu}_{s}\right) \equiv \min _{\vec{\mu}_{b}}\left[\sum_{j=1}^{\# \text { of bins }}\left(\frac{O_{j}-P_{j}\left(\vec{\mu}_{b}, \vec{\mu}_{s}\right)}{\sigma_{j}}\right)^{2}\right]+\text { const },
$$

where $O_{j}$ ("number of observed events") denotes the number of events in the $j$-th bin without lensing, and $P_{j}\left(\vec{\mu}_{b}, \vec{\mu}_{s}\right)$ ("number of predicted events") denotes the number of events expected if there is a lens. $\sigma_{j}$ is the uncertainty associated with the $j$-th bin, which is given by adding in quadrature the statistical uncertainty $\sqrt{P_{j}\left(\vec{\mu}_{b}, \vec{\mu}_{s}\right)}$ and a systematic uncertainty. The latter accounts for quasi-random deviations of the source spectrum from the model, and we assume its magnitude to be $5 \%$. (We will also show how our results change if the systematic

\footnotetext{
${ }^{7}$ The power law model is not strongly motivated physically, and is just used here as a cross check to verify the robustness of our predictions. In particular, if there is an exponential cutoff in the GRB spectrum, it is expected at an energy much higher than $150 \mathrm{keV}$ [56].

${ }^{8}$ Note that our statistical procedure differs from the one in [27]. Our approach combines the likelihood of the lensed signal for every possible 3-dimensional position of the lens, weighted by the probability of finding a lens at a given position. Ref. [27], in contrast, investigates the observability of lensing as a function of the lens mass and the transverse position of the source, but without fully taking into account the dependence on the redshift of the lens $z_{L}$.
} 


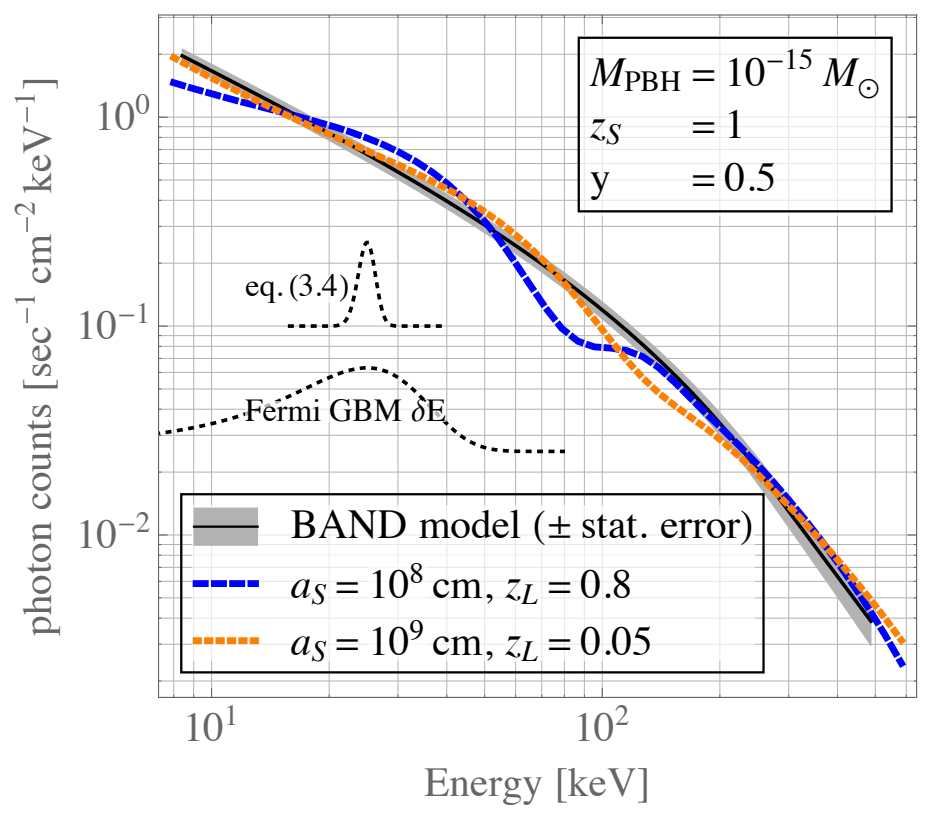

Figure 4. Simulated GRB spectra based on the BAND model with parameters $A=$ 14.08 counts $\mathrm{sec}^{-1} \mathrm{~cm}^{-2} \mathrm{keV}^{-1}, E_{0}=160 \mathrm{keV}, \alpha_{1}=-0.9$, and $\alpha_{2}=-2.5$. We compare the unlensed spectrum (black) to the predicted spectra in the presence of a $\mathrm{PBH}$ lens with mass $M=10^{-15} M_{\odot}$ and impact parameter $y=0.5$. Wave optics effects as well as the finite size of the GRB emission region are taken into account, where for the latter we use either $a_{S}=10^{8} \mathrm{~cm}$ (blue) or $10^{9} \mathrm{~cm}$ (orange). The source is assumed to be located at redshift $z_{S}=1$. For highly optimistic (small) $a_{S}$, a pronounced interference pattern is visible almost independently of the position of the lens, while for more realistic (larger) $a_{S}$, only a lens rather close to the observer may lead to an observable effect. The gray band drawn around the unlensed data represents the statistical uncertainty. The black dotted gaussians show the detector response assuming the FERMI energy resolution as well as the resolution of eq. (3.4) that we optimistically assume for future detectors. The signal that one observes in the detector would be a convolution of the detector response with the line simulated GRB spectrum.

uncertainty is set to $0 \%$ or $10 \%$ instead.) The vector $\vec{\mu}_{b}$ contains the relevant parameters of the background model (four for the BAND and BKN models, three for the power law model with exponential cutoff). To be conservative, we minimize over $\vec{\mu}_{b}$, i.e. we choose the background parameters that best fit the data. The vector $\vec{\mu}_{s}$ contains the parameters of the lens, namely its mass $M$, its redshift $z_{L}$, and its normalized impact parameter in the lens plane $y=\beta / \theta_{E}$. Thus, $L_{0}$ compares the unlensed spectrum to the lensed spectrum for fixed lens and source parameters. Note that we can use Gaussian rather than Poisson statistics here because the number of photon events per bin is large.

We can define a lensing cross section $\sigma\left(D_{L}\right)=\pi\left(y_{\max } \theta_{E} D_{L}\right)^{2}$, where $y_{\max }$ is the maximal normalized distance from the lens to observer-source line of sight that still leads to a sizable lensing signal. (A sizable signal is defined as a signal that can be distinguished, at a given confidence level (CL) from an unlensed signal.) In other words, $y_{\max }$ is obtained by solving

$$
-2 \log \left(\frac{L_{0}\left(\vec{\mu}_{s} \mid y=y_{\max }\right)}{L_{0}\left(\vec{\mu}_{s} \mid y=\infty\right)}\right)=\alpha,
$$

where $\alpha$ is the quantile one of the $\chi^{2}$ distribution with one degree of freedom corresponding 
to the chosen CL. For instance, $\alpha=2.7$ for $90 \%$ CL and $\alpha=9$ for $3 \sigma$ CL. The optical depth $\tau$ of the source is obtained as

$$
\tau=\int_{0}^{z_{S}} \frac{d z_{L}}{H\left(z_{L}\right)} \sigma\left(D_{L}\right) \frac{\rho_{\mathrm{PBH}}}{M}\left(1+z_{L}\right)^{2},
$$

where $\rho_{\mathrm{PBH}} / M$ is the number density of lenses at the present epoch. We integrate over the redshift of the lens, which is equivalent, up to the Hubble constant $H\left(z_{L}\right)$, to integrating over comoving distance. The factor $\left(1+z_{L}\right)^{2}$ appears as a combination of a factor $\left(1+z_{L}\right)^{3}$ that accounts for the increase in lens density with redshift and a factor $\left(1+z_{L}\right)^{-1}$ needed to convert between the physical and comoving longitudinal coordinate.

To obtain limits on the density of lenses, we need to take into account the dependence of $L_{0}$ on $\vec{\mu}_{s}$. To this end, we define the overall likelihood for lensing of a single GRB source with size $a_{S}$ and redshift $z_{S}$ according to

$$
L^{1-\mathrm{GRB}}\left(M, \rho_{\mathrm{PBH}}, a_{S}, z_{S}\right) \equiv L_{0}(0)+\int d^{3} x \frac{\rho_{\mathrm{PBH}}}{M}\left(1+z_{L}\right)^{3}\left[L_{0}\left(\vec{\mu}_{s}\right)-L_{0}(0)\right] .
$$

Here, the integral runs over physical (not comoving) coordinates, and the factor $\left(1+z_{L}\right)^{3}$ once again takes into account the increase of the lens density with redshift. The integration region extends from the observer to the source in the longitudinal direction, and out to infinity in the transverse directions. We here make the (realistic) assumption that the probability of a single GRB being lensed by multiple compact DM objects is $\ll 1$.

Of course, the sensitivity can be significantly boosted by observing not a single GRB, but many of them. For $n$ observed GRBs, the total likelihood is

$$
L^{n-\mathrm{GRBs}}\left(M, \rho_{\mathrm{PBH}},\left\{a_{S, k}\right\},\left\{z_{S, k}\right\}\right) \equiv \prod_{k=1}^{n} L^{1-\mathrm{GRB}}\left(M, \rho_{\mathrm{PBH}}, h_{S, k}, z_{S, k}\right),
$$

where $a_{S, k}$ and $z_{S, k}$ denote the size and redshift of the $k$-th GRB.

We set $95 \%$ CL limits on the mass and density of the compact DM objects by equating the log-likelihood ratio with the $95 \%$ quantile of the $\chi^{2}$ distribution with two degrees of freedom $\left(M\right.$ and $\left.\rho_{\mathrm{PBH}}\right)$ :

$$
-2 \log \left(\frac{L^{n-\mathrm{GRBs}}\left(M, \rho_{\mathrm{PBH}},\left\{a_{S, k}\right\},\left\{z_{S, k}\right\}\right)}{L^{n-\mathrm{GRBs}}\left(0,0,\left\{a_{S, k}\right\},\left\{z_{S, k}\right\}\right)}\right)=5.99 \quad(95 \% \mathrm{CL}) .
$$

Here, the denominator corresponds to the likelihood of the data in the absence of any lensing.

\subsection{Results}

With the above likelihood formalism in hand, we can now study the sensitivity of current and future data to compact DM objects. We focus on the mass range $10^{-17} M_{\odot}$ to $10^{-14} M_{\odot}$. This mass window is motivated by the requirement that a sizable phase difference between the different lensed images of the source should occur within the energy range of GRB spectra. Our results are shown in fig. 5. We see that with 20 GRBs with well-measured redshifts (the number of GRBs used in [27]), a meaningful limit can only be set if the GRBs are assumed to be point-like, i.e. $a_{S} \lesssim 10^{8} \mathrm{~cm}$. Here, by "meaningful limit" we mean a limit that constrains the cosmological PBH abundance, $\Omega_{\mathrm{PBH}}$, to be less than the total DM abundance in the Universe, $\Omega_{\mathrm{DM}}$. As argued in section 2.3 , the assumption $a_{S} \lesssim 10^{8} \mathrm{~cm}$ is almost certainly 


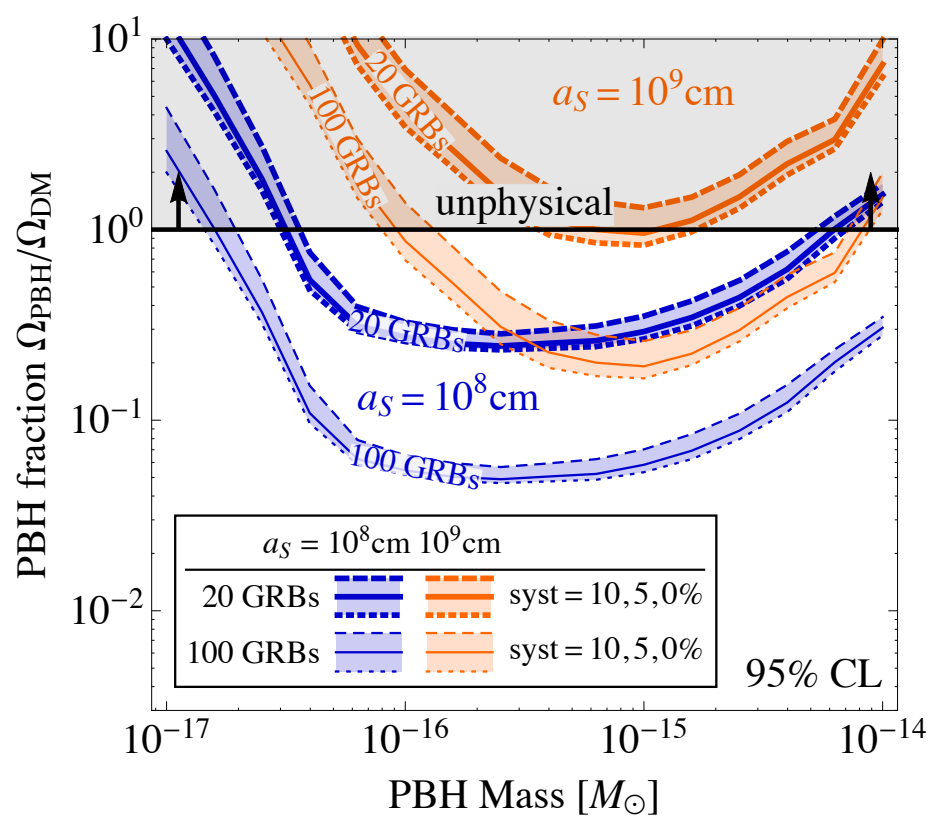

Figure 5. Sensitivity of femtolensing searches to the primordial black hole contribution $\Omega_{\mathrm{PBH}} / \Omega_{\mathrm{DM}}$ to the overall DM density in the Universe. We show the projected sensitivity for different assumptions on the number of suitable GRBs with well-measured redshifts in the data sample. We also illustrate the dependence on the size $a_{S}$ of the emission region of a typical GRB, where $a_{S}=10^{8} \mathrm{~cm}$ should be considered a highly optimistic value, and $a_{S}=10^{9} \mathrm{~cm}$ an optimistic but possible value. The colored bands indicate the impact of systematic effects (uncorrelated random fluctuations in each bin). We use the BAND model for the GRB spectrum throughout. For other spectral models, limits would change by not more than a few tens of percent. We have assumed the redshift of all GRBs in the sample to be $z_{S}=1$.

overly optimistic. If $a_{S}$ is only one order of magnitude larger (which is still very optimistic), no limit can be set.

This may change in the future if the available sample of GRBs is significantly extended. With 100 GRBs, sensitivity to an $\mathcal{O}(30 \%)$ fraction of PBHs can be achieved for $a_{S} \sim 10^{9} \mathrm{~cm}$. The sensitivity would improve to $\Omega_{\mathrm{PBH}} / \Omega_{\mathrm{DM}} \sim 0.06$ if $a_{S} \sim 10^{8} \mathrm{~cm}$. Our conclusions are essentially independent of the choice of GRB model (BAND vs. BKN vs. power law with exponential cutoff). They depend somewhat on the assumed systematic uncertainty, with the sensitivity deteriorating by at most a factor of two if the assumed systematic error is increased from $0 \%$ to $10 \%$. Note that we have very conservatively assumed systematic errors to be completely uncorrelated between energy bins.

In fig. 6, we put our projected constraints into a broader context by comparing to other limits on PBHs. We see that future femtolensing constraints, albeit weak, may cover a mass range that is otherwise inaccessible and where viable PBH DM could exist.

While the projected limits shown in figs. 5 and 6 apply only to PBH DM, we can use the arguments given at the end of section 2.4 as a starting point for estimating also the sensitivity to other compact DM structures. For UCMHs, we have argued above that the interference fringes are shifted to higher energies compared to the $\mathrm{PBH}$ case, with the magnitude of the shift, $m\left(\theta_{0}\right) / M_{\text {cusp }}$, given by eqs. (2.29) and (2.30) for UCMHs with $\rho(r) \propto\left(r_{0} / r\right)^{9 / 4}$. We 


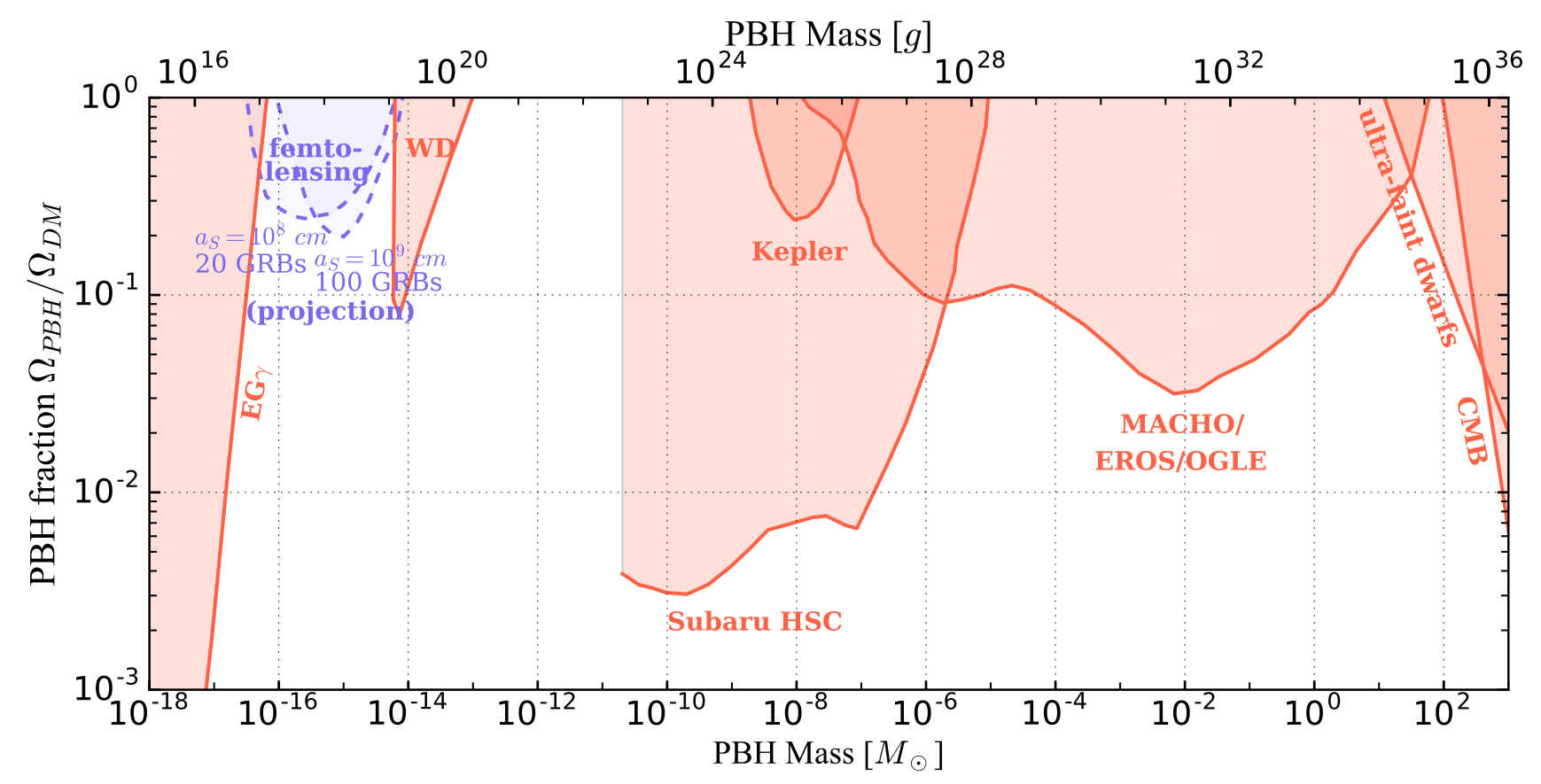

Figure 6. Future femtolensing sensitivity to primordial black holes compared to other probes. In particular, we compare our projected limits (blue dashed contours) to limits based on extragalactic background photons $(\mathrm{EG} \gamma)$ from $\mathrm{PBH}$ evaporation [13], from the non-destruction of white dwarfs (WD) [18], from microlensing searches by Subaru HSC [4], Kepler [58], MACHO [1], EROS [2], and OGLE [3], from the dynamics of ultra-faint dwarf galaxies [59], and from CMB anisotropies due to accretion onto PBHs [60]. (Stronger CMB limits are obtained if more aggressive assumptions on accretion by PBHs are adopted [61].) The Subaru HSC limits are cut off at $M \sim 10^{-11} M_{\odot}$ because below that mass, the geometric optics approximation employed in ref. [4] is not valid. We also do not include neutron star limits [15] because of their dependence on controversial assumptions about the DM density in globular clusters. We have taken the limits shown here from the compilation in ref. [36]. In computing our projected limits, we have assumed the redshift of all GRBs in the sample to be $z_{S}=1$, we have used the BAND model for the GRB spectrum, and we have assumed a $5 \%$ systematic uncertainty, uncorrelated between energy bins.

therefore estimate that the projected femtolensing exclusion limits on such UCMHs will be similar in shape to the ones for PBHs if the PBH mass is understood as the "equivalent black hole mass" $m\left(\theta_{0}\right)$ from eqs. (2.29) and (2.30). Expressed in terms of $M_{\text {cusp }}$, the limits on UCMHs are thus shifted to higher masses by a factor $M_{\text {cusp }} / m\left(\theta_{0}\right)$ compared to the limits shown in figs. 5 and 6 . We moreover need to take into account the fact that the DM number density scales inversely with mass, hence we expect the projected exclusion curves to also move upwards by a factor $M_{\text {cusp }} / m\left(\theta_{0}\right)$, corresponding to a weakening of the limit by that factor. In view of this, and given that we have seen how difficult it will already be to constrain PBH DM using femtolensing, we refrain from a more detailed sensitivity study for UCMHs.

\section{Conclusions}

To summarize, we have critically investigated the potential of gravitational femtolensing to constrain compact DM structures such as primordial black holes or ultracompact DM miniha- 
los. Femtolensing exploits the tiny time delay between the multiple lensed images of a distant source. Interference between the images leads to characteristic fringes in the energy spectrum of observed photons, see fig. 4. As sources, we consider in particular gamma ray bursts at $\mathcal{O}(1)$ redshift. These sources are most easily observed at energies $\gtrsim 10 \mathrm{keV}$, hence observable interference pattern require time delays $\lesssim 10^{-19}$ sec. This means that the best sensitivity is expected for compact DM objects in the mass range from $10^{-17}$ to $10^{-14} M_{\odot}$.

We have argued that the simple geometric picture of femtolensing based on point-like sources and lenses that is often used in the literature is not appropriate in reality. First, it is not true that photons travel from the source to the detector along one of just two discrete paths. In fact, when the time delay becomes comparable to the inverse photon frequency (which for point-like lenses is equivalent to the photon wave length becoming comparable to the Schwarzschild radius of the lens), wave optics effects become non-negligible. It is then necessary to integrate the photon amplitude over the whole lens plane. This leads to $\mathcal{O}(1)$ corrections to the interference pattern at the lower end of the photon energy spectrum. Second, while the approximation of a point-like lens works for primordial black holes, it is not satisfied for ultra-compact mini-halos, and even less so for NFW-like structures. We have therefore computed femtolensing effects for generic power-law density profiles, and have explicitly shown numerical results for the self-similar infall profile with $\rho(r) \propto r^{-9 / 4}$.

The most important correction in femtolensing of GRBs is coming from the non-negligible size $a_{S}$ of the GRB source itself. In fact, we have argued that a GRB could only be treated as point-like for the purpose of femtolensing if the photon emission region was smaller than $a_{S} \sim 10^{8} \mathrm{~cm}$. And while estimates for the size of the emission region can vary by a few orders of magnitude, $a_{S} \sim 10^{8} \mathrm{~cm}$ seems unrealistically small. For more realistic assumptions on the value of $a_{S} \gtrsim 10^{10} \mathrm{~cm}$, the femtolensing effect is almost entirely washed out. This means that, contrary to previous claims, current GRB data is insufficient to constrain compact DM structures such as primordial black holes, even if their abundance $\Omega_{\mathrm{PBH}}$ saturates the observed DM abundance $\Omega_{\mathrm{DM}}$ in the Universe. We have, however, demonstrated that constraints down to $\Omega_{\mathrm{PBH}} / \Omega_{\mathrm{DM}} \sim 0.2$ would become possible with a sample of about 100 observed GRBs with well-measured redshifts and spectra, and with small $a_{S}$. Such GRBs are expected to be characterized by very fast intrinsic variability at sub-millisecond time scales.

Since femtolensing constraints on compact DM objects are still out of reach we conclude that there are currently no firm bounds on PBH DM in the mass range of $10^{-17} \div 10^{-11}$ (with the exception of a small wedge of parameter space excluded by white dwarf observation). This is illustrated in fig. 6 . While several ideas has been put forward to constrain this mass range, for instance picolensing [25] and the capture of PBHs by stars or neutron stars, none of these methods has yielded decisive bounds until now, so this region of parameter space still awaits exploration.

\section{Acknowledgments}

We are grateful to Yacine Ali-Haïmoud, Juan Garcia Bellido, Kfir Blum, Lam Hui and Kathryn Zurek for useful discussions. JK has been supported by the German Research Foundation (DFG) under Grant Grant Nos. KO 4820/11, FOR 2239, EXC-1098 (PRISMA) and by the European Research Council (ERC) under the European Union's Horizon 2020 research and innovation programme (grant agreement No. 637506, " $\nu$ Directions"). JK would also like to thank CERN for hospitality and support. 


\section{A The size of the prompt emission region in GRBs}

In this appendix we discuss the transverse size of the $\gamma$-ray emitting regions in GRBs, which, as we have seen in section 2.3, plays a crucial role in the study of femtolensing. We will review estimates for the size of the emission region based on measurements of the variability time scale, and we will also discuss the lower bound following from the required transparency of the emission region to $\gamma$-rays.

It is believed that the prompt $\gamma$-emission of GRBs is produced by electrons and positrons accelerated in relativistic shock waves. The non-thermal $\gamma$-ray spectrum implies that the emission region must be optically thin. To reconcile this requirement with the observed energetics of the bursts, the bulk Lorentz factor of the $\gamma$-ray emitting material must be large, $\Gamma \gtrsim 100[62]$.

We etsimate the transverse size of the emission region following the same approach as Ref. [29]. Consider a blob of material of size $a_{S}$ moving with velocity $v$ at an angle $\theta_{\text {obs }}$ to the observer's line of sight. In the rest frame of the blob the minimal variability time scale of the emission is simply given by the light-crossing time,

$$
\hat{t}_{\mathrm{var}} \sim a_{S} / c .
$$

The observed variability time $t_{\mathrm{var}}$ is related to $\hat{t}_{\mathrm{var}}$ by the relativistic Doppler formula,

$$
t_{\mathrm{var}}=\left(1+z_{S}\right)\left(1-\frac{v}{c} \cos \theta_{\mathrm{obs}}\right) \Gamma \hat{t}_{\mathrm{var}},
$$

where $\Gamma \equiv\left(1-(v / c)^{2}\right)^{-1 / 2}$ and we have taken into account the cosmological redshift of the source. Due to the relativistic beaming effect, we have $\theta_{\text {obs }} \sim 1 / \Gamma$. Combining this with eqs. (A.1) and (A.2) one obtains the estimate

$$
a_{S} \sim \frac{c \Gamma t_{\mathrm{var}}}{1+z_{S}} \simeq \frac{10^{11} \mathrm{~cm}}{1+z_{S}} \times\left(\frac{t_{\mathrm{var}}}{0.03 \mathrm{sec}}\right)\left(\frac{\Gamma}{100}\right)
$$

The minimal variability time scales for various GRBs have been determined in ref. [42]. They lie within the range $t_{\mathrm{var}}^{\mathrm{sGRB}} \sim(0.01 \div 0.1)$ sec for short GRBs and $t_{\mathrm{var}}^{\mathrm{lGRB}} \sim(0.1 \div 1)$ sec for the long ones. These results are consistent with the earlier estimates of ref. [29] that give average variability time scales $t_{\mathrm{var}}$ of $0.036 \mathrm{sec}$ and $1.2 \mathrm{sec}$ for short and long GRBs, respectively. We see that for a typical short GRB with $z \sim 1, t_{\mathrm{var}} \sim 0.03 \mathrm{sec}$, and $\Gamma \sim \mathcal{O}(100)$, the transverse size is,

$$
a_{S} \sim 10^{11} \mathrm{~cm} .
$$

For long GRBs this estimate becomes an order of magnitude larger. ${ }^{9}$

In the above estimates we adopted the standard picture of a GRB with a relativistic jet of $\gamma$-ray emitting material pointing towards the observer. One may wonder how the reasoning is modified in the case of off-axis observation, like in the recent GRB 170817A

\footnotetext{
${ }^{9}$ Note that Ref. [42] derives also the distribution of the emission radii $R_{\mathrm{em}}$, i.e. the distance from the GRB central engine at which $\gamma$-rays are emitted. The central value of this distribution is $R_{\mathrm{em}} \sim 3 \times 10^{13} \mathrm{~cm}$ $\left(10^{14} \mathrm{~cm}\right)$ for short (long) GRBs. Due to relativistic beaming, the transverse size of the patch visible by an observer on Earth is related to this distance as $a_{S} \sim R_{\mathrm{em}} / \Gamma$. Assuming $\Gamma \sim \mathcal{O}(100)$, this again gives $a_{S} \sim 10^{11} \mathrm{~cm}\left(10^{12} \mathrm{~cm}\right)$ for short (long) GRBs.
} 
[63, 64] accompanied by the gravitational wave event GW170817 [65], which has confirmed the identification of neutron star mergers as progenitors of short GRBs. As discussed in [66], the observational data pertaining to this event across the electromagnetic spectrum are best described by a model in which a mildly relativistic wide-angle shock with $\Gamma \sim 2.5$ breaks off the ambient material and emits $\gamma$-rays at a distance $R_{\mathrm{em}} \simeq 2.4 \times 10^{11} \mathrm{~cm}$ from the central engine. In this case relativistic beaming is practically absent, so $a_{S}$ is comparable to $R_{\mathrm{em}}$. We again recover the estimate (A.4).

If all GRBs satisfy the estimate (A.4), observing any femtolensing in their spectra will be essentially hopeless. So let us ask how robust this estimate is. A hint that there may be GRBs with smaller sizes is already provided by the analysis of Ref. [42], which finds that about $10 \%$ of the bursts (both short and long) exhibit faster variability, $t_{\mathrm{var}} \lesssim 2 \times 10^{-3}$ sec, which leads to an estimated size $a_{S} \lesssim 10^{10} \mathrm{~cm}$. Thus, one can speculate that some rare GRBs at the tail of the distribution could have $t_{\text {var }}$ yet another order of magnitude shorter, bringing their sizes close to the values of $a_{S}$ required for efficient femtolensing. It is also worth pointing out that the determination of the minimal variability time scale is affected by instrumental systematics, such as the detector sensitivity and the light-curve sampling. Thus, it might happen, in principle, that some of the measured variability time scales overestimate the true intrinsic variability time scale of the source.

An alternative method for constraining $a_{S}$ is based on the requirement that the emission region must be optically thin. Two processes can lead to absorption of $\gamma$-rays: production of $e^{+} e^{-}$pairs in $\gamma \gamma$-scattering, and Compton scattering on electrons and positrons. Let us start with the first process.

Consider a photon with energy $\hat{E}$ in the rest frame of the emitting blob. The optical depth for pair production is,

$$
\tau_{\gamma \gamma}(\hat{E})=a_{S} \int_{\hat{\epsilon}_{\mathrm{th}}} d \hat{\epsilon} \sigma_{\gamma \gamma}(\hat{\epsilon}) \hat{n}_{\gamma}(\hat{\epsilon})
$$

where $\sigma_{\gamma \gamma}(\hat{\epsilon})$ is the (angular-averaged) cross section of collision with an ambient photon of energy $\hat{\epsilon}, \hat{n}_{\gamma}(\hat{\epsilon})$ is the spectral density of such photons, and

$$
\hat{\epsilon}_{\mathrm{th}}=\frac{m_{e}^{2} c^{4}}{\hat{E}}
$$

is the threshold energy for pair production. To estimate $\hat{n}_{\gamma}(\hat{E})$ we notice that the number of photons with energy $\hat{E}$ emitted by the blob during its proper time $d \hat{t}$ is

$$
d N \simeq c a_{S}^{2} \hat{n}_{\gamma}(\hat{E}) d \hat{E} d \hat{t} .
$$

This is related to the photon flux $f(E)$ seen by the observer at energy $E=\Gamma \hat{E} /\left(1+z_{S}\right)$ as

$$
d N \simeq \frac{d_{S}^{2}}{\Gamma^{2}\left(1+z_{S}\right)^{2}} f(E) d E d t,
$$

where $d_{S}$ is the luminosity distance of the GRB and the factor $1 / \Gamma^{2}$ is due to the fact that in the observer's frame the emission is beamed into a narrow cone with opening angle $\sim \Gamma^{-1}$. Next, due to the Doppler effect we have (cf. eq. (A.2)) $d t \simeq\left(1+z_{S}\right) d \hat{t} \Gamma$. Inserting all these relations into (A.5) we obtain,

$$
\tau_{\gamma \gamma}(\hat{E}) \simeq \frac{d_{S}^{2}}{c a_{S} \Gamma^{2}\left(1+z_{S}\right)^{2}} \int_{\hat{\epsilon}_{\mathrm{th}}} d \hat{\epsilon} \sigma_{\gamma \gamma}(\hat{\epsilon}) f\left(\Gamma \hat{\epsilon} /\left(1+z_{S}\right)\right) .
$$


Following common practice, we now assume that the spectrum of the prompt emission is described by a power law,

$$
f(E)=A E^{\alpha} .
$$

with spectral index $\alpha$ close to -2 . Then the integral in eq. (A.9) can be evaluated with the result,

$$
\tau_{\gamma \gamma}(\hat{E}) \simeq \frac{d_{S}^{2} \sigma_{T}}{c a_{S}} \eta(\alpha) A \Gamma^{\alpha-2}\left(1+z_{S}\right)^{-\alpha-2}\left(\frac{m_{e}^{2} c^{4}}{\hat{E}}\right)^{\alpha+1}
$$

where $\sigma_{T}$ is the Thomson cross section and the numerical coefficient is $[67,68]$

$$
\eta(\alpha)=\frac{3(\alpha(\alpha-5)(\alpha-1)-2) \sqrt{\pi} \Gamma(-\alpha)}{8 \alpha(1-\alpha)(\alpha-2)(\alpha-1) \Gamma(3 / 2-\alpha)} .
$$

Expressing the optical depth in terms of the photon energy measured by the observer we arrive at,

$$
\tau_{\gamma \gamma}(E) \simeq \frac{d_{S}^{2} \sigma_{T}}{c a_{S}} \eta(\alpha) \Gamma^{2 \alpha-1}\left(1+z_{S}\right)^{-2 \alpha-3} \frac{m_{e}^{2} c^{4}}{E} f\left(\frac{m_{e}^{2} c^{4}}{E}\right),
$$

Up to a numerical coefficient of order one, this coincides with the expression obtained in [69]. For $\alpha<-1$ the optical depth grows with the energy of the photon. Requiring that it is smaller than one for photons with the maximal observed energy $E_{\max }$ we obtain,

$$
a_{S}>2.5 \times 10^{6} \mathrm{~cm} \times\left(\frac{d_{S}}{7 \mathrm{Gpc}}\right)^{2}\left(\frac{f_{500}}{10^{-3} \mathrm{sec}^{-1} \mathrm{~cm}^{-2} \mathrm{keV}^{-1}}\right)\left(\frac{E_{\max }}{1 \mathrm{MeV}}\right)\left(\frac{\Gamma}{1000}\right)^{-5},
$$

where $f_{500}$ is the $\gamma$-ray flux at $500 \mathrm{keV}$ and we have assumed $\alpha=-2, z_{S}=1$ for the numerical estimate.

Due to instrumental limitations the spectra of most GRBs are measured up to $E_{\max } \sim$ $1 \mathrm{MeV}$. This corresponds to the energy $\hat{E} \sim\left(1+z_{S}\right) \Gamma^{-1} \mathrm{MeV} \ll 1 \mathrm{MeV}$ in the blob rest frame. If the spectrum were cut at these energies, all the photons would be below the threshold of pair production and we would not have to worry about absorption at all. However, it is believed that the spectrum of a typical GRB extends to much higher energies. This is supported by detection of high energy emission (up to $\sim 100 \mathrm{GeV}$ ) from several GRBs [56]. When such observations are available, the corresponding maximal energy can be used in (A.14) to set a strong lower bound on the source size.

We now turn to absorption due to Compton scattering of $\gamma$-photons on electrons and positrons. We will consider $e^{ \pm}$created by the process of $\gamma \gamma$ scattering discussed above. As will be seen shortly, the resulting constraint is stronger than (A.14) in the case when a direct measurement of the high-energy component of $\gamma$-radiation is absent [69].

Assuming, for simplicity, that electrons are non-relativistic in the rest frame of the emitting material, the optical depth for photons with $\hat{E} \lesssim m_{e} c^{2}$ is essentially independent of their energy,

$$
\tau_{\gamma e} \simeq a_{S} \sigma_{T} \hat{n}_{e}
$$

where $\hat{n}_{e}$ is the total density of electrons. The latter is estimated as the density of photons for which the blob is optically thick with respect to pair production,

$$
\hat{n}_{e}=\int_{\hat{\epsilon}_{\mathrm{cr}}} d \hat{\epsilon} \hat{n}_{\gamma}(\hat{\epsilon}),
$$


where $\hat{\epsilon}_{\mathrm{cr}}$ is determined from the equation $\tau_{\gamma \gamma}\left(\hat{\epsilon}_{\mathrm{cr}}\right)=1$. Using the expression (A.11) we obtain,

$$
\tau_{\gamma e} \simeq \frac{\eta(\alpha)}{-\alpha-1}\left[\frac{d_{S}^{2} \sigma_{T}}{c a_{S}} A \Gamma^{\alpha-2}\left(1+z_{S}\right)^{-\alpha-2}\left(m_{e} c^{2}\right)^{\alpha+1}\right]^{2} .
$$

Requiring that the emission region be optically thin, $\tau_{\gamma e}<1$, translates into

$$
a_{S}>\left(\frac{\eta(\alpha)}{-\alpha-1}\right)^{1 / 2} \frac{d_{S}^{2} \sigma_{T}}{c} \Gamma^{\alpha-2}\left(1+z_{S}\right)^{-\alpha-2} m_{e} c^{2} f\left(m_{e} c^{2}\right) .
$$

For the numerical values $\alpha=-2, z_{S}=1$ this yields,

$$
a_{S}>1.8 \times 10^{9}\left(\frac{d_{S}}{7 \mathrm{Gpc}}\right)^{2}\left(\frac{f_{500}}{10^{-3} \mathrm{sec}^{-1} \mathrm{~cm}^{-2} \mathrm{keV}^{-1}}\right)\left(\frac{\Gamma}{1000}\right)^{-4} \mathrm{~cm} .
$$

We see that for extreme values of the boost factor $\Gamma \sim 1200$ which may occur in some GRBs [70] the lower bound on the source size is quite small. Of course, it would be too optimistic to interpret this lower bound as a plausible value of $a_{S}$. On the other hand, the above derivation is only an order-of-magnitude estimate. Moreover, it relies on a power-law extrapolation of the GRB spectrum. As such, it shows that the possibility for some GRBs to have sizes $a_{S} \lesssim 10^{9} \mathrm{~cm}$ is not completely excluded. Further progress in our quantitative understanding of the physics of GRBs is required to conclude if this option is viable or not. Note that according to eq. (A.3), if such GRBs exist, they are expected to have variablity at sub-millisecond time scale.

\section{References}

[1] MACHO collaboration, R. A. Allsman et al., MACHO project limits on black hole dark matter in the 1-30 solar mass range, Astrophys. J. 550 (2001) L169 [astro-ph/0011506].

[2] EROS-2 collaboration, P. Tisserand et al., Limits on the Macho Content of the Galactic Halo from the EROS-2 Survey of the Magellanic Clouds, Astron. Astrophys. 469 (2007) 387 [astro-ph/0607207].

[3] L. Wyrzykowski et al., The OGLE View of Microlensing towards the Magellanic Clouds. IV. OGLE-III SMC Data and Final Conclusions on MACHOs, Mon. Not. Roy. Astron. Soc. 416 (2011) 2949 [1106.2925].

[4] H. Niikura, M. Takada, N. Yasuda, R. H. Lupton, T. Sumi, S. More et al., Microlensing constraints on primordial black holes with the Subaru/HSC Andromeda observation, 1701.02151.

[5] B. J. Carr and S. W. Hawking, Black holes in the early Universe, Mon. Not. Roy. Astron. Soc. 168 (1974) 399.

[6] S. Clesse and J. García-Bellido, Massive Primordial Black Holes from Hybrid Inflation as Dark Matter and the seeds of Galaxies, Phys. Rev. D92 (2015) 023524 [1501.07565].

[7] B. Carr, F. Kuhnel and M. Sandstad, Primordial Black Holes as Dark Matter, Phys. Rev. D94 (2016) 083504 [1607.06077].

[8] C. J. Hogan and M. J. Rees, AXION MINICLUSTERS, Phys. Lett. B205 (1988) 228.

[9] E. W. Kolb and I. I. Tkachev, Axion miniclusters and Bose stars, Phys. Rev. Lett. 71 (1993) 3051 [hep-ph/9303313]. 
[10] E. W. Kolb and I. I. Tkachev, Nonlinear axion dynamics and formation of cosmological pseudosolitons, Phys. Rev. D49 (1994) 5040 [astro-ph/9311037].

[11] E. Hardy, Miniclusters in the Axiverse, JHEP 02 (2017) 046 [1609.00208].

[12] M. Sasaki, T. Suyama, T. Tanaka and S. Yokoyama, Primordial black holes-perspectives in gravitational wave astronomy, Class. Quant. Grav. 35 (2018) 063001 [1801.05235].

[13] B. J. Carr, K. Kohri, Y. Sendouda and J. Yokoyama, New cosmological constraints on primordial black holes, Phys. Rev. D81 (2010) 104019 [0912.5297].

[14] F. Capela, M. Pshirkov and P. Tinyakov, Constraints on Primordial Black Holes as Dark Matter Candidates from Star Formation, Phys. Rev. D87 (2013) 023507 [1209.6021].

[15] F. Capela, M. Pshirkov and P. Tinyakov, Constraints on primordial black holes as dark matter candidates from capture by neutron stars, Phys. Rev. D87 (2013) 123524 [1301.4984].

[16] P. Pani and A. Loeb, Tidal capture of a primordial black hole by a neutron star: implications for constraints on dark matter, JCAP 1406 (2014) 026 [1401.3025].

[17] F. Capela, M. Pshirkov and P. Tinyakov, A comment on "Exclusion of the remaining mass window for primordial black holes ...", arXiv:1401.3025, 1402.4671.

[18] P. W. Graham, S. Rajendran and J. Varela, Dark Matter Triggers of Supernovae, Phys. Rev. D92 (2015) 063007 [1505.04444].

[19] G. Defillon, E. Granet, P. Tinyakov and M. H. G. Tytgat, Tidal capture of primordial black holes by neutron stars, Phys. Rev. D90 (2014) 103522 [1409.0469].

[20] C. Conroy, A. Loeb and D. Spergel, Evidence Against Dark Matter Halos Surrounding the Globular Clusters MGC1 and NGC 2419, Astrophys. J. 741 (2011) 72 [1010.5783].

[21] R. Ibata, C. Nipoti, A. Sollima, M. Bellazzini, S. Chapman and E. Dalessandro, Do globular clusters possess Dark Matter halos? A case study in NGC 2419, Mon. Not. Roy. Astron. Soc. 428 (2013) 3648 [1210.7787].

[22] S. Naoz and R. Narayan, Globular Clusters and Dark Satellite Galaxies through the Stream Velocity, Astrophys. J. 791 (2014) L8 [1407.3795].

[23] C. Popa, S. Naoz, F. Marinacci and M. Vogelsberger, Gas rich and gas poor structures through the stream velocity effect, Mon. Not. Roy. Astron. Soc. 460 (2016) 1625 [1512.06862].

[24] A. Gould, Femto microlensing of gamma-ray bursters, Astrophys. J. Lett. 386 (1992) L5.

[25] R. J. Nemiroff and A. Gould, Probing for MACHOs of mass 10( $\left.{ }^{* *}-15\right)$-solar-mass 10**-7-solar-mass with gamma-ray burst parallax spacecraft, Astrophys. J. 452 (1995) L111 [astro-ph/9505019].

[26] G. F. Marani, R. J. Nemiroff, J. P. Norris, K. Hurley and J. T. Bonnell, Gravitationally lensed gamma-ray bursts as probes of dark compact objects, Astrophys. J. 512 (1999) L13 [astro-ph/9810391].

[27] A. Barnacka, J. F. Glicenstein and R. Moderski, New constraints on primordial black holes abundance from femtolensing of gamma-ray bursts, Phys. Rev. D86 (2012) 043001 [1204.2056].

[28] S. Davidson and T. Schwetz, Rotating Drops of Axion Dark Matter, Phys. Rev. D93 (2016) 123509 [1603.04249].

[29] A. Barnacka and A. Loeb, A size-duration trend for gamma-ray burst progenitors, Astrophys. J. 794 (2014) L8 [1409.1232].

[30] K. Z. Stanek, B. Paczynski and J. Goodman, Features in the Spectra of Gamma-Ray Bursts, Astrophys. J. 413 (1993) L7.

[31] N. Matsunaga and K. Yamamoto, The finite source size effect and the wave optics in gravitational lensing, JCAP 0601 (2006) 023 [astro-ph/0601701]. 
[32] E. W. Kolb and I. I. Tkachev, Femtolensing and picolensing by axion miniclusters, Astrophys. J. 460 (1996) L25 [astro-ph/9510043].

[33] K. M. Zurek, C. J. Hogan and T. R. Quinn, Astrophysical Effects of Scalar Dark Matter Miniclusters, Phys. Rev. D75 (2007) 043511 [astro-ph/0607341].

[34] A. Ulmer and J. Goodman, Femtolensing: Beyond the semiclassical approximation, Astrophys. J. 442 (1995) 67 [astro-ph/9406042].

[35] M. Bartelmann, Gravitational Lensing, Class. Quant. Grav. 27 (2010) 233001 [1010.3829].

[36] K. Inomata, M. Kawasaki, K. Mukaida and T. T. Yanagida, Double inflation as a single origin of primordial black holes for all dark matter and LIGO observations, Phys. Rev. D97 (2018) 043514 [1711.06129].

[37] R. Takahashi and T. Nakamura, Wave effects in gravitational lensing of gravitational waves from chirping binaries, Astrophys. J. 595 (2003) 1039 [astro-ph/0305055].

[38] S. Jung and C. S. Shin, Gravitational-Wave Lensing Fringes by Compact Dark Matter at LIGO, 1712.01396.

[39] P. Christian, S. Vitale and A. Loeb, Detecting Stellar Lensing of Gravitational Waves with Ground-Based Observatories, 1802.02586.

[40] S.-S. Li, S. Mao, Y. Zhao and Y. Lu, Gravitational lensing of gravitational waves: A statistical perspective, 1802.05089.

[41] T. T. Nakamura and S. Deguchi, Wave Optics in Gravitational Lensing, Progress of Theoretical Physics Supplement 133 (1999) 137.

[42] V. Z. Golkhou, N. R. Butler and O. M. Littlejohns, The Energy-Dependence of GRB Minimum Variability Timescales, Astrophys. J. 811 (2015) 93 [1501.05948].

[43] J. Enander, A. Pargner and T. Schwetz, Axion minicluster power spectrum and mass function, JCAP 1712 (2017) 038 [1708.04466].

[44] P. Tinyakov, I. Tkachev and K. Zioutas, Tidal streams from axion miniclusters and direct axion searches, JCAP 1601 (2016) 035 [1512.02884].

[45] Y. Bai, V. Barger and J. Berger, Hydrogen Axion Star: Metallic Hydrogen Bound to a QCD Axion BEC, JHEP 12 (2016) 127 [1612.00438].

[46] M. Fairbairn, D. J. E. Marsh and J. Quevillon, Searching for the QCD Axion with Gravitational Microlensing, Phys. Rev. Lett. 119 (2017) 021101 [1701.04787].

[47] M. Fairbairn, D. J. E. Marsh, J. Quevillon and S. Rozier, Structure formation and microlensing with axion miniclusters, Phys. Rev. D97 (2018) 083502 [1707.03310].

[48] J. A. Fillmore and P. Goldreich, Self-similiar gravitational collapse in an expanding universe, Astrophys. J. 281 (1984) 1.

[49] E. Bertschinger, Self - similar secondary infall and accretion in an Einstein-de Sitter universe, Astrophys. J. Suppl. 58 (1985) 39.

[50] M. Vogelsberger, S. D. M. White, R. Mohayaee and V. Springel, Caustics in growing Cold Dark Matter Haloes, Mon. Not. Roy. Astron. Soc. 400 (2009) 2174 [0906.4341].

[51] M. Vogelsberger, R. Mohayaee and S. D. M. White, Nonspherical similarity solutions for dark halo formation, Mon. Not. Roy. Astron. Soc. 414 (2011) 3044 [1007.4195].

[52] M. S. Delos, A. L. Erickcek, A. P. Bailey and M. A. Alvarez, Are ultracompact minihalos really ultracompact?, Phys. Rev. D97 (2018) 041303 [1712.05421].

[53] T. Bringmann, P. Scott and Y. Akrami, Improved constraints on the primordial power spectrum at small scales from ultracompact minihalos, Phys. Rev. D85 (2012) 125027 [1110.2484]. 
[54] D. Band et al., BATSE observations of gamma-ray burst spectra. 1. Spectral diversity., Astrophys. J. 413 (1993) 281.

[55] P. N. Bhat et al., The Third Fermi gbm Gamma-ray Burst Catalog: the First six Years, Astrophys. J. Suppl. 223 (2016) 28 [1603.07612].

[56] L. Nava, High-energy emission from Gamma-Ray Bursts, 1804.01524.

[57] C. Meegan et al., The Fermi Gamma-Ray Burst Monitor, Astrophys. J. 702 (2009) 791 [0908.0450].

[58] K. Griest, A. M. Cieplak and M. J. Lehner, New Limits on Primordial Black Hole Dark Matter from an Analysis of Kepler Source Microlensing Data, Phys. Rev. Lett. 111 (2013) 181302.

[59] T. D. Brandt, Constraints on MACHO Dark Matter from Compact Stellar Systems in Ultra-Faint Dwarf Galaxies, Astrophys. J. 824 (2016) L31 [1605.03665].

[60] Y. Ali-Haimoud and M. Kamionkowski, Cosmic microwave background limits on accreting primordial black holes, Phys. Rev. D95 (2017) 043534 [1612.05644].

[61] V. Poulin, P. D. Serpico, F. Calore, S. Clesse and K. Kohri, CMB bounds on disk-accreting massive primordial black holes, Phys. Rev. D96 (2017) 083524 [1707.04206].

[62] T. Piran, The physics of gamma-ray bursts, Rev. Mod. Phys. 76 (2004) 1143 [astro-ph/0405503].

[63] A. Goldstein et al., An Ordinary Short Gamma-Ray Burst with Extraordinary Implications: Fermi-GBM Detection of GRB 170817A, Astrophys. J. 848 (2017) L14 [1710. 05446].

[64] V. Savchenko et al., INTEGRAL Detection of the First Prompt Gamma-Ray Signal Coincident with the Gravitational-wave Event GW170817, Astrophys. J. 848 (2017) L15 [1710.05449].

[65] Virgo, LiGO Scientific collaboration, B. Abbott et al., GW170817: Observation of Gravitational Waves from a Binary Neutron Star Inspiral, Phys. Rev. Lett. 119 (2017) 161101 [1710.05832].

[66] M. M. Kasliwal et al., Illuminating Gravitational Waves: A Concordant Picture of Photons from a Neutron Star Merger, Science 358 (2017) 1559 [1710.05436].

[67] R. J. Gould and G. P. Schreder, Pair Production in Photon-Photon Collisions, Phys. Rev. 155 (1967) 1404.

[68] R. Svensson, Non-thermal pair production in compact X-ray sources - First-order Compton cascades in soft radiation fields, Mon. Not. Roy. Astron. Soc. 227 (1987) 403.

[69] Y. Lithwick and R. Sari, Lower Limits on Lorentz Factors in Gamma-Ray Bursts, Astrophys. J. 555 (2001) 540 [astro-ph/0011508].

[70] J. L. Racusin, S. R. Oates, P. Schady, D. N. Burrows, M. de Pasquale, D. Donato et al., Fermi and Swift Gamma-ray Burst Afterglow Population Studies, Astrophys. J. 738 (2011) 138 [1106.2469]. 